\title{
Study on Defects, Composition and Microstructure of Electron Beam Cold Hearth Melting Titanium Ingot
}

\author{
Qingzhu Sun ${ }^{1,2, a}$, Haibo Wang ${ }^{1, b}$, Qi Lai ${ }^{2, c}$, Xueyuan Jin ${ }^{1}$, Shuanghua Huang ${ }^{2}$ \\ ${ }^{1}$ School of Materials Engineering,Panzhihua University, Panzhihua 617000, China \\ ${ }^{2}$ Panxi Science and Technology Innovation Center, Panzhihua University, Panzhihua 617000, \\ ahaerbinsqz@163.com, bimsdxwhb@163.com, ㄷ49129834@qq.com
}

Keywords: Electron Beam Cold Hearth Melting, titanium, defects, composition. Abstract. In this paper, the composition, defects and microstructures of titanium ingots prepared by Electron Beam Cold Hearth Melting are studied. The results show that the defects of the titanium ingot are mainly distributed in the final solidified position, The microstructure is mainly composed of dense columnar dendrites $\alpha$ phase and a small amount of $\beta$ phase distributed on the grain boundary, the organization is small and uniform. Electron Beam Cold Hearth Melting is a good method to impurity, the content of impurity element of the ingot after a smelting are all below the standard .

\section{Introduction}

Known as the rise of the "third metal" ,titanium has a series of excellent characteristics, such as small density, high strength, high specific strength, excellent corrosion resistance, good heat resistance, low temperature performance,non-magnetic, thermal conductivity, Low anti-damping performance and low elastic modulus. Tensile strength and yield strength are very close.It has broad application prospects in the advanced construction, aerospace, chemical metallurgy, energy and power, shipbuilding, construction, sports supplies [1]. Electron Beam Cold Hearth Melting (EBCHM) was developed in the 1960s, after nearly 50 years of development, it has occupied a very important position in the production of high-quality titanium and titanium ingot [2]. Compared with the traditional VAR, when titanium or titanium alloy is melted by the Electron Beam Cold Hearth Melting, the melting speed and melting temperature can be flexibly controlled, This is not only conducive to provide sufficient dissolution conditions to remove the high and low density inclusions of titanium and titanium alloy, but also effective to control the ingot element evaporation , segregation and other issues. Therefore, high density and low density inclusions can be well eliminated[3]. Because of its unique advantage in the removal of inclusions, Electron Beam Cold Hearth Melting has been used to produce high-quality titanium and titanium alloy ingot, and thus it is widly concerned and applicated by the aero engine manufacturers and titanium producers[4]. In this paper, a pure titanium ingot with columnar crystal structure was obtained by Electron Beam Cold Hearth Melting, and the composition, defects and microstructure of titanium ingot were studied.

\section{Test materials and methods}

Test materials .The titanium sponge, which was removed the oxidized sponge titanium and foreign matter,was placed in a copper water-cooled crucible.Titanium dioxide was heated and melted by the electron beam, it was injected into a crystallizer and cooled to obtain pure titanium ingot. Titanium Ingot is as shown in Fig. 1, the size of titanium ingot is $100 \mathrm{~mm}$ in diameter and $400 \mathrm{~mm}$ in length.

Performance characterization methods.The Oxide of the pure titanium ingot was removed, it was firstly tested by nondestructive testing. The chemical composition of pure titanium ingot was analyzed, and the microstructure of pure titanium ingot was observed by optical microscope. 


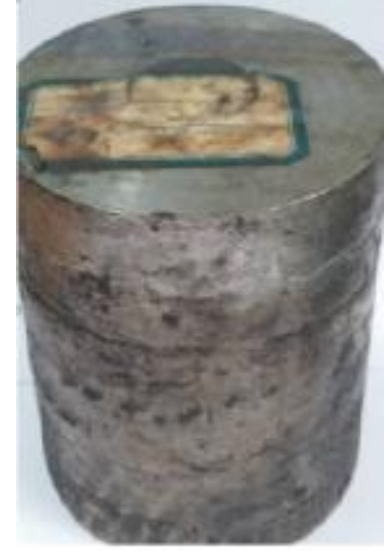

Fig. 1 Titanium Ingot preparaed by EBCHM

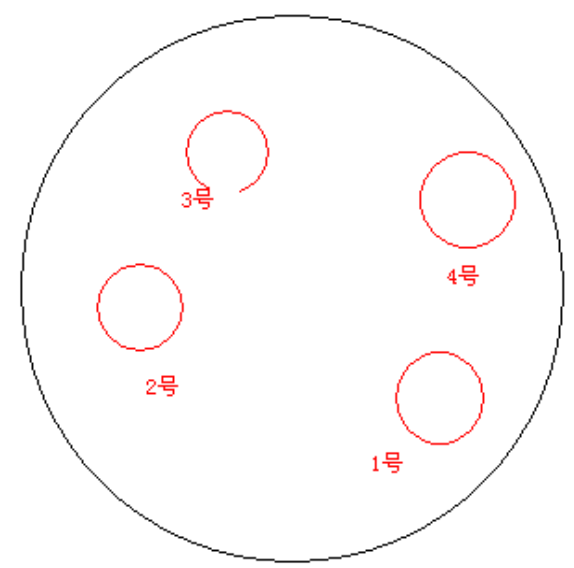

Fig. 2 Diagram of the abnormal position

\section{Experimental results and discussion}

Analysis of nondestructive testing results. The location and type of defects were analyzed by UTL620 digital ultrasonic flaw detector,whose straight probe was $\oint 20$, ultrasonic frequency was $2.50 \mathrm{MHz}$. the detection plane was the upper surface, and the defect reflection waves were detected at the four positions shown in Fig.2. The appearance of the four positions, in which the wave can be detected, were mainly in the middle columnar crystal area. The four nondestructive detection waves are respectively shown as Fig. 3, Fig. 4, Fig. 5 and Fig. 6. From the depth of the waves, the defects can be seen in the final position of the ignot, other locations less.that is indicated that the density of the titanium ingot is good, but it is defective at the last end. This phenomenon is consistent with the principle of solidification, during the solidification process, solute and defects offen accumulate in

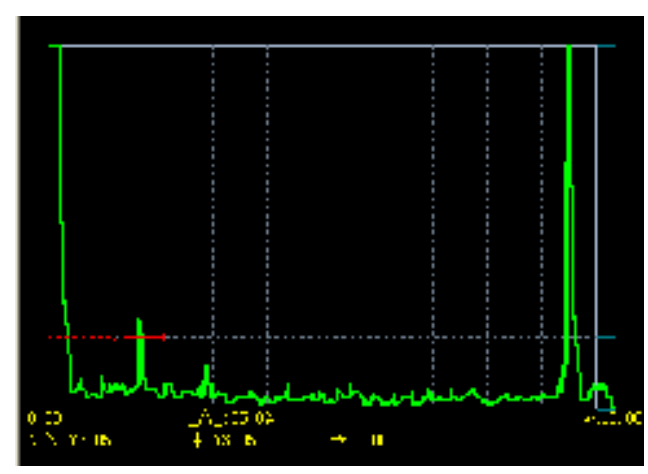

Fig.3 Nondestructive detection wave at position 1

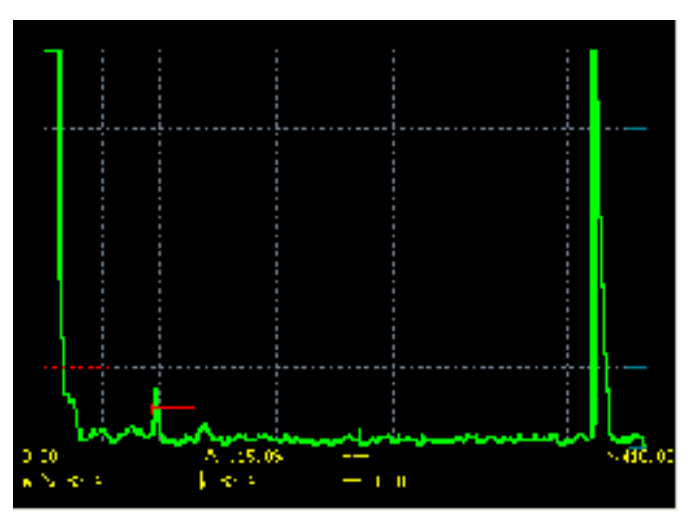

Fig.5 Nondestructive detection wave at position3

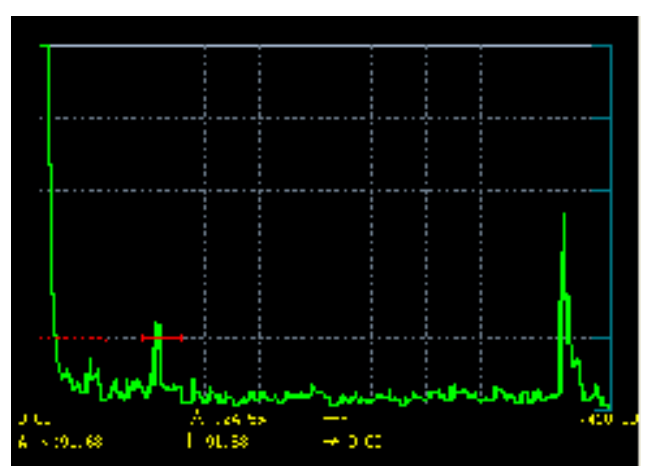

Fig.4 Nondestructive detection wave at position 2

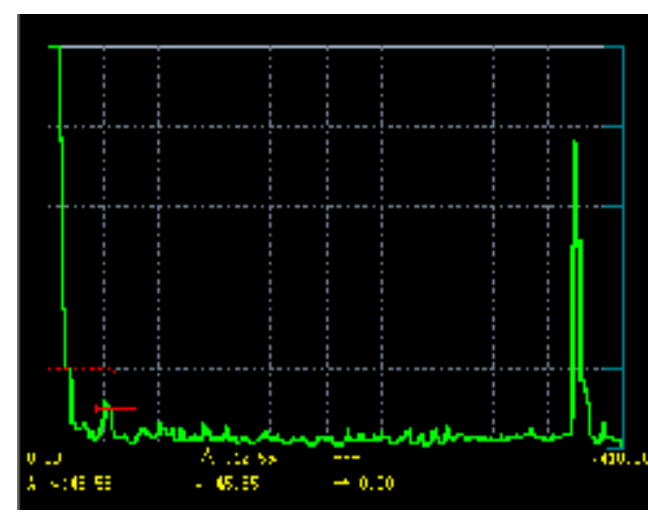

Fig.6 Nondestructive detection wave at position 4 
the front of the solid-liquid interface, consequently, solute and defects are concentration distribution in the final solidification.

Component analysis.Titanium and its alloys are highly chemically active metals that react fairly readily in the liquid state with oxygen, nitrogen, hydrogen and carbon, They are present in titanium in the form of $\mathrm{TiO}, \mathrm{TiN}, \mathrm{TiC}$ [3]. So the removal of impurities is one of the main task of electron beam cold hearth melting titanium and titanium alloy.

In this paper, the components and impurity elements of titanium ingots were detected. Because of the low content of impurity elements, it is not easy to detect the specific content. Therefore, qualitative method such as XRD and quantitative methods such as energy spectrum analysis and chemical analysis were used .

The preparation process of the test sample for XRD performance was as follows, the pure titanium ingot was cut in the middle of the length, the two specimens cut from solidification initial were defined as 1-1, 1-2, and the four samples cut from solidification end were defined as 2-1, 2-2, 2-3 and 2-4. Fig. 7 and Fig. 8 show that the composition of the solidification initiation and solidification ends of the ingot is mainly pure titanium, through qualitatively analyze, the samples are without carbon, hydrogen, oxygen, nitrogen and also their compounds .

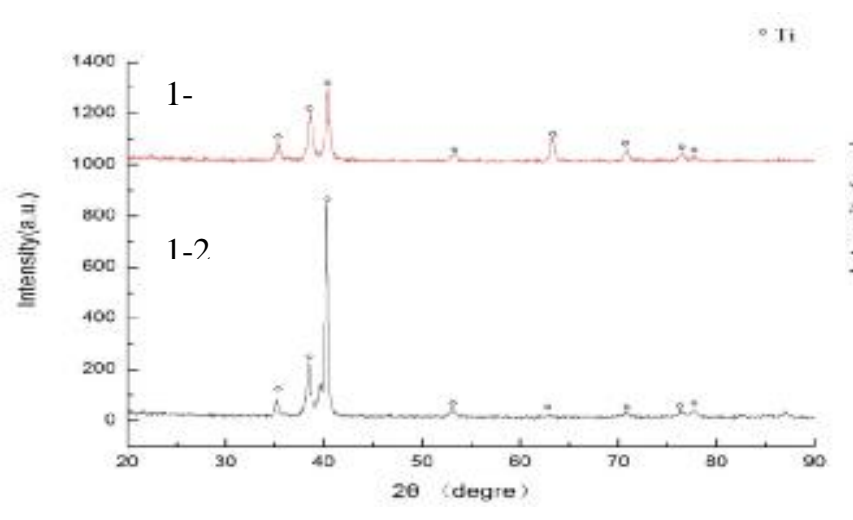

Fig.7 XRD patterns of solidification initiation

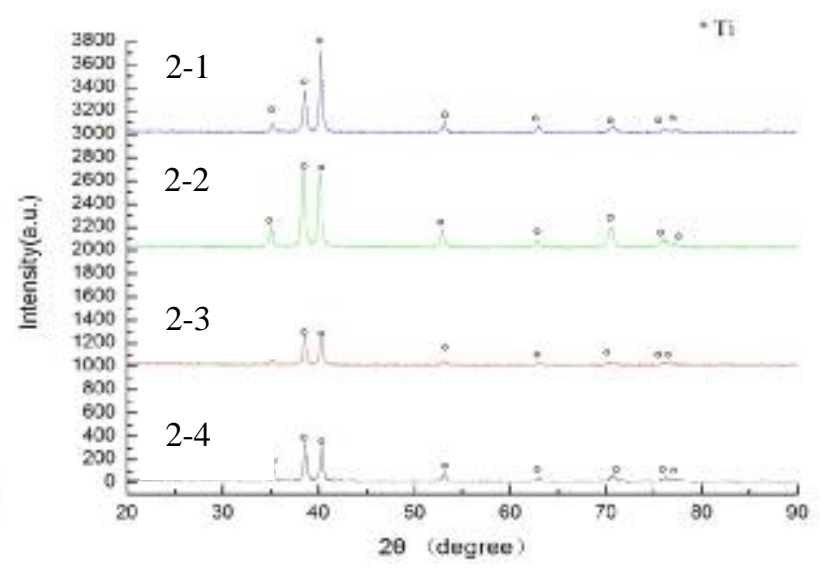

Fig. 8 XRD patterns of solidification end

The datas of SEM energy spectrum of sample 1-1 and sample 2-1 are shown as table 1 and table 2.The results show that the main components in ingot are titanium, and the contents of carbon, hydrogen, oxygen and nitrogen are not detected. This is consistent with the XRD results, which proves that the purity of pure titanium ingot is high.

Table 1 SEM energy spectrum datas of the solidifying front sample 1-1

\begin{tabular}{ccc}
\hline element & Weight percentage[\%] & Atomic percentage \\
\hline Ti & 100.00 & 100.00 \\
others & 0.00 & 0.00 \\
Total amount & 100.00 & \\
\hline Table 2 & SEM energy & spectrum datas of the solidifying front sample 2-1 \\
\hline element & Weight percentage[\%] & Atomic percentage \\
\hline Ti & 100.00 & 100.00 \\
others & 0.00 & 0.00 \\
Total amount & 100.00 &
\end{tabular}

The impurity elements were detected by chemical analysis. In Table 3, impurity element content is very small, can be ignored, in full compliance with GB / T3620.1 standard.

Metallographic results and analysis. The microstructure of the ingot is shown in Fig.9. As shown in Fig. 9a, tissue density of sample 1-1 is good, $\alpha$ phase is the matrix, which is columnar dendrites, the number of $\beta$ phase is less, it is spherical and small particles, distributed in the boundary 
Table 3 Test Data Of Impurity Element Chemical Analysis

\begin{tabular}{ccccc}
\hline element & $\mathrm{O}$ & $\mathrm{N}$ & $\mathrm{C}$ & $\mathrm{H}$ \\
\hline MAX & 0.01 & 0.013 & 0.011 & 0.001 \\
MIN & 0 & 0 & 0 & 0 \\
R & 0.005 & 0.006 & 0.006 & 0.0005 \\
\hline
\end{tabular}

of $\alpha$ phase. Fig. 9b is the microstructure of sample 2-1. Tissue density is good too, the size of $\beta$ phase is bigger than that of sample 1-1, the number of $\beta$ phase is more than that of sample 1-1. Due to a small amount of defects in the ingot, in the erosion and cleaning process, a small part of microstructure was blurred.
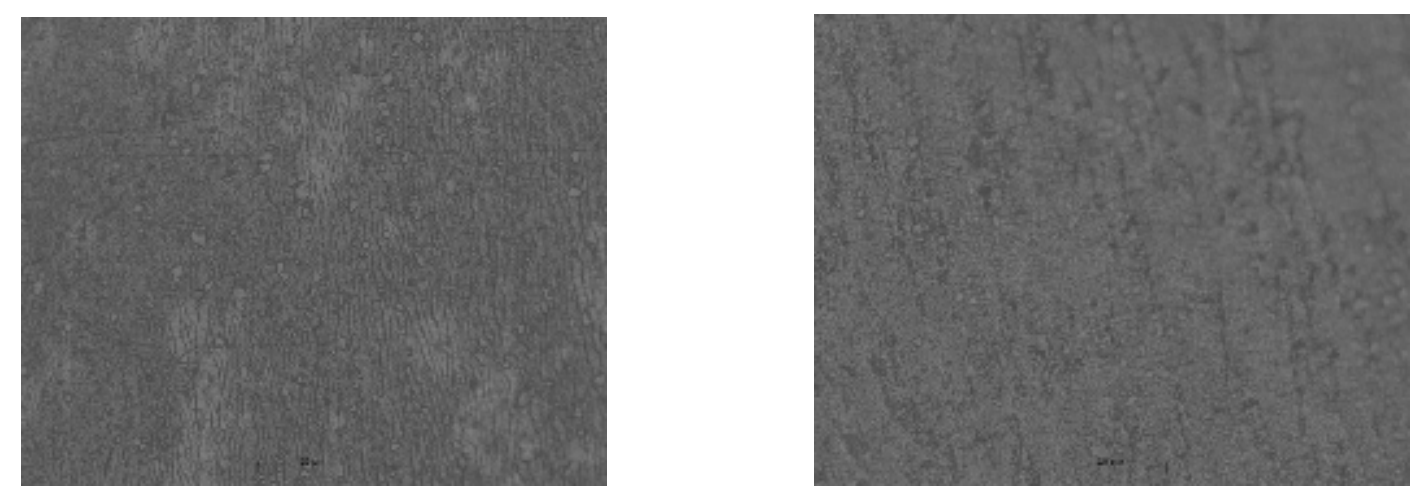

a. microstructure of solidification initiation 1-1 b. microstructure of solidification end 2-1

Fig.9 The microstructure of the ingot 1000X

\section{Conclusions}

1)there are some defects at pure titanium ingot produced by EBCHM,the defects are mainly distributed in the final solidification position, ingot tissue is dense and uniform.

2) EBCHM is good technology to remove impurities.After single melted by EBCHM, impurity element content of ingots was below the standard content, high-quality titanium ingot was produced. 3 ) The microstructure of ignot is mainly composed of dense columnar dendrites $\alpha$ phase and a small amount of $\beta$ phase distributed on the $\alpha$ phase grain boundary, the organization is small.

\section{Acknowledgements}

This work was financially supported by key science and technology projects of Panzhihua.

\section{References}

[1] Duan Junwei. Estreicher:Nonferrous Metals Processing. Forum Vol. $56-57$ (2011) , p. 40.

[2] Ma Rongbao, Chen Feng, Guo Bin . Estreicher:Titanium Industry Process. Forum Vol. 37-40(2008), p.25.

[3] Luo Lei, Yang Guanjun, MaoXiaonan, et al. Estreicher:Hot Working Technology. Forum Vol. 56-59(2009), p.38.

[4] Yu Lanlan, Mao Xiaonan, Zhang Yingming,et al. Estreicher:Titanium Industry Process. .Forum Vol.14-18(2009), p.26 